\title{
Regulation of a Servo Piezo Mechanical Hydraulic Actuator for Intake Valves in Camless Combustion Engines
}

\author{
PAOLO MERCORELLI \\ Leuphana University of Lueneburg \\ Institute of Product and Process Innovation \\ Universitaetsallee 1, D-21335 Lueneburg
}

GERMANY

\begin{abstract}
In this paper a hybrid actuator is proposed. The hybrid actuator consists of a piezo mechanical structure and a hydraulic ratio displacement. Particular attention is paid to a liquid spring model of the displacement ratio which represents the hydraulic part of the mechanism. The proposed mechanism represents the fundamental part of a more complex one which consists of a supplementary hydraulic part to control intake valves of a combustion engine. The whole mechanism is controlled using a cascade repetitive control combined with a proportional derivative (PD) controller and a feed-forward action to track a periodic signal. Measured results of the closed loop carriedoutthesuitabilityofthecontrolapproach.
\end{abstract}

Key-Words: Hybrid actuators, modelling, repetitive control, engines

Received: November 27, 2019. Revised: March 31, 2020. Accepted: April 29, 2020. Published: May 2, 2020.

\section{Introduction}

The idea which this paper presents is to use a hybrid actuator composed by a piezo, mechanical and hydraulic parts. Moreover, piezo actuators present in general less problems of electromagnetic compatibility due to the quasi-absence of the inductance effects. In fact, the piezoelectric actuator (PA) has been used in precise positioning applications such as, for instance, atomic force microscopy [1], [2]. The main advantages of PA are nanometer scale, high stiffness, and a fast response. However, since PA has a nonlinear property which is called hysteresis effect, it leads to inaccuracy in positioning control with a high precise performance. Very recent literature indicates a clear interest in applications of piezo in mechatronic systems. In particular, for oscillations rejection and soft landing recently piezo actuator were utilised. In [4] a novel active vibration control strategy using piezoelectric actuators for metrological devices affected by low external loads is proposed. The control strategy combines a classical sky-hook feedback with a feed-forward control. The effect of hysteresis is minimized by compensating the sensitivity variations of the actuator in oscillatory movements. As already mentioned soft landing and more in general velocity control are important issues. In [5] the velocity control of a large-range piezoelectrically actuated (piezo) stepper is analysed. In [6] a self-sensing technique for piezoelectric actuators used in precise positioning applications like micromanipulation and

E-ISSN: 2224-3429 microassembly is presented. The main novelty is that both displacement and force signals can be simultaneously estimated. This allows a feedback control using one of these two signals with a display of the other signal. In many technical applications periodical signals are used and to correct the steady-state error a repetitive control can be taken into consideration. It is known that the repetitive control is based on the principle of the internal model according to [7]. In order to control periodic reference variables or for compensating periodic disturbances a repetitive control as in [8] is applied. The internal model principle is also applied both for continuous and for discrete systems. In addition, an unstable controlled system must be first stabilized using an additional controller. Repetitive control has a significant advantage that is very easy to be parameterized without transfer function of the controlled system. A crucial part of the actuator consists of a stroke displacement ratio with an oil chamber and because of possible faults of the sealings the right functioning of this part of the actuator can be compromised. To conclude, the main contribution of this paper consists of:

- presenting a new conception and modelling of a hybrid actuator

- proposing a repetitive controller together with an adaptive feed-forward controller.

Figure 1 shows the diagram of the positions of an engine intake and exhaust valves. In this figure the

Volume 15, 2020 

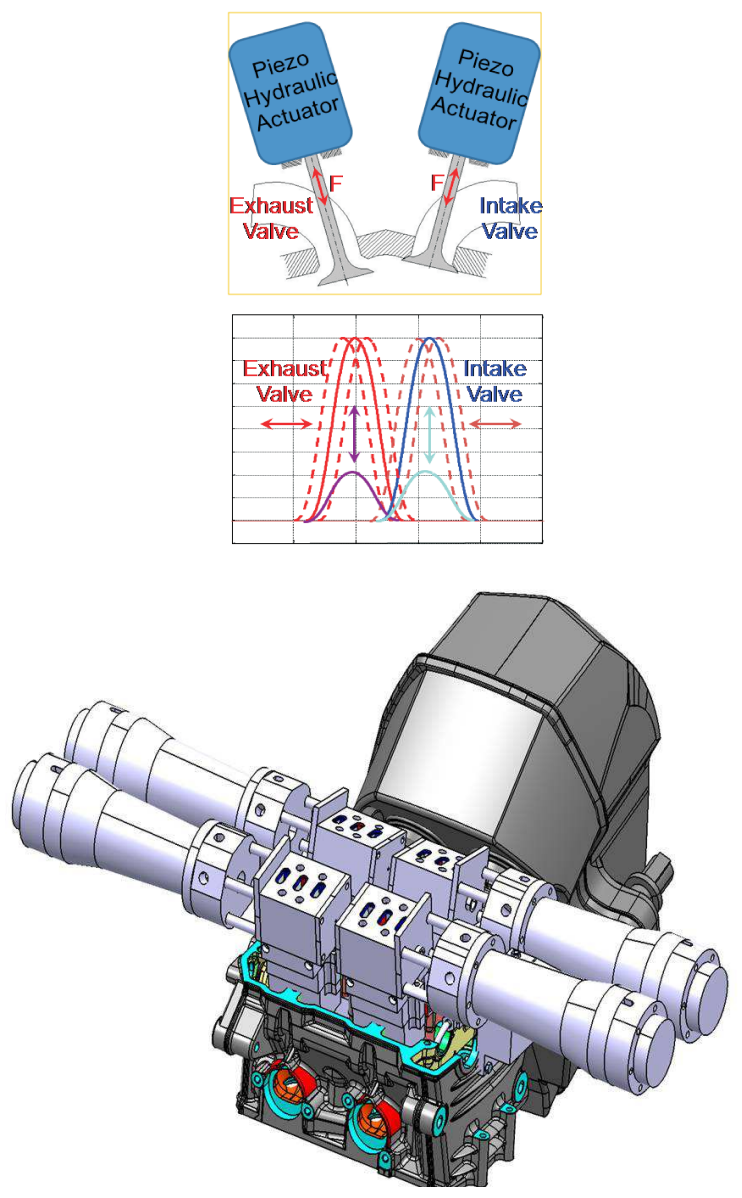

Figure 1: New structure of the engine

intake and the exhaust valve position profiles are indicated. Moreover, Fig. 1 shows the new engine structure with, evidently, four piezo actuators. An overview on the complete hybrid actuator is depicted in Fig. 2. The paper is organised in the following way. Section 2 indicates a possible model of the hybrid actuator. In Section 4 the proposed control scheme consisting of an adaptive feed-forward action based on a fault detection is presented. Measured results together with the conclusion close the paper.

$K_{F L}:$ stiffness constant

$K_{F L 1}$ : stiffness constant related to a large surface of the stroke ratio

$K_{F L 2}$ : stiffness constant related to a small surface of the stroke ratio

$A_{1}$ : large surface of the stroke ratio

$A_{2}$ : small surface of the stroke ratio

$V_{\text {in }}(t)$ : piezo input voltage

$V_{z}(t)$ : active piezo input voltage

$K_{x}$ : internal piezo stiffness constant

$D_{x}:$ piezo factor

$D_{x} K_{x}=T_{e m}:$ transformer ratio

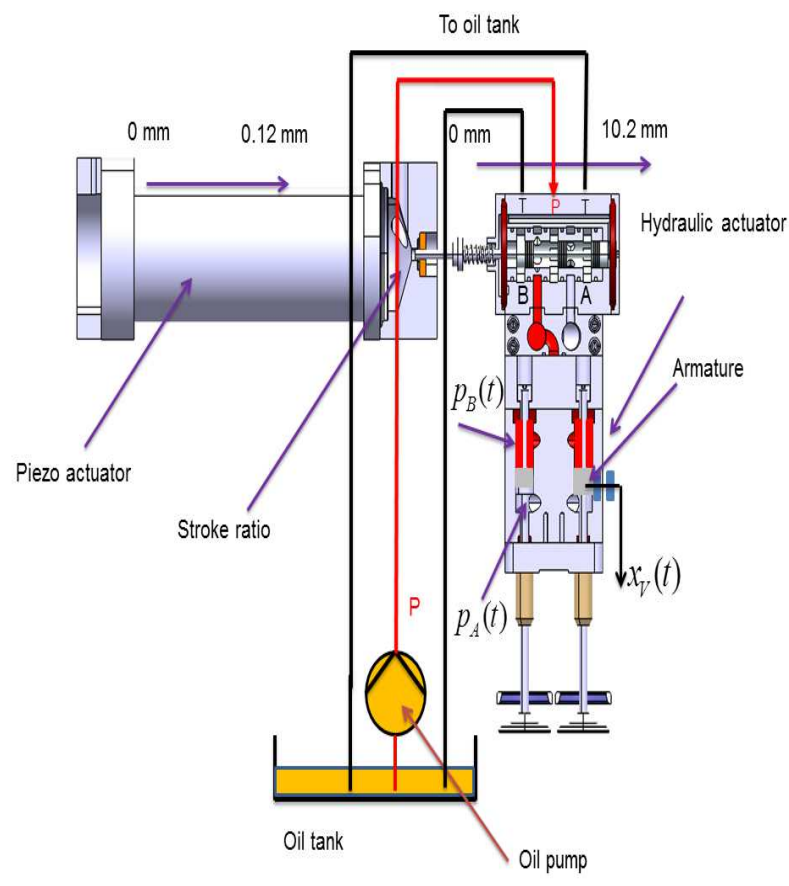

Figure 2: Model of the whole actuator

$F_{z}(t)=V_{z}(t) D_{x} K_{x}:$ motor piezo force

$m_{S K}$ : mass of the servo piston

$M_{v}$ : mass of the servo valve

$x_{1}(t)$ : piezo position

$x_{2}(t)$ : servo piston position

$x_{2 d}(t)=V_{z}(t) D_{x}$ : motor piezo movement

$x_{V}(t)$ : valve piston position

$K_{S K}$ : servo piston spring constant

$D_{S K}$ : damping factor of the servo piston spring

$V_{M}$ : steady-state factor of the mechanical system

$V_{H}$ : steady-state factor of the hydraulic system

$p(t)$ : pressure

$Q_{t h}$ : volumetric flow

$A_{V P}:$ surface of the servo piston

$A_{L}$ : leakage flow parameter

\section{Model of the Hybrid Actuator}

The mathematical model is the same which is adopted in [9] and in [10]; in this part of the paper the model of the actuator is presented again. The actuator consists of different technical parts: a piezoelectric structure, a servo piston structure and a hydraulic one.

\subsection{Piezoelectric Structure}

The mathematic model is the same which is adopted in [11]. The details on this model can be found in [12]. Considering the whole system described in Fig. 
3 with the assumption of compressibility of the oil, the whole mechanical system can be represented by a spring mass structure as shown in the conceptual scheme of Fig. 4. Concerning the piezo actuator, observing Fig. 4, $K$ and $D$ represent the elasticity and the friction constant of the spring which is antagonist to the piezo effect and is incorporated in the PEA. In the technical literature, factor $D_{x} K_{x}=T_{e m}$ is known with the name "transformer ratio" and states that the most important characteristic of the electromechanical transducer in which $K_{x}$ is the elasticity constant factor of the PEA and $D_{x}$ is the parameter which is responsible to transform voltage into movement. In fact, another well-known physical relation is $F_{z}(t)=$ $D_{x} K_{x} V_{z}(t)$ which represents the piezo force in which $V_{z}$ is the internal voltage. In the ideal case, we have that $V_{z}(t)=V_{i n}(t)$ where $V_{i n}(t)$ states the input voltage. According to [12], in Fig. 3 and in two schemes which represent the considered actuator are reported.

\subsection{Servo Piston Structure}

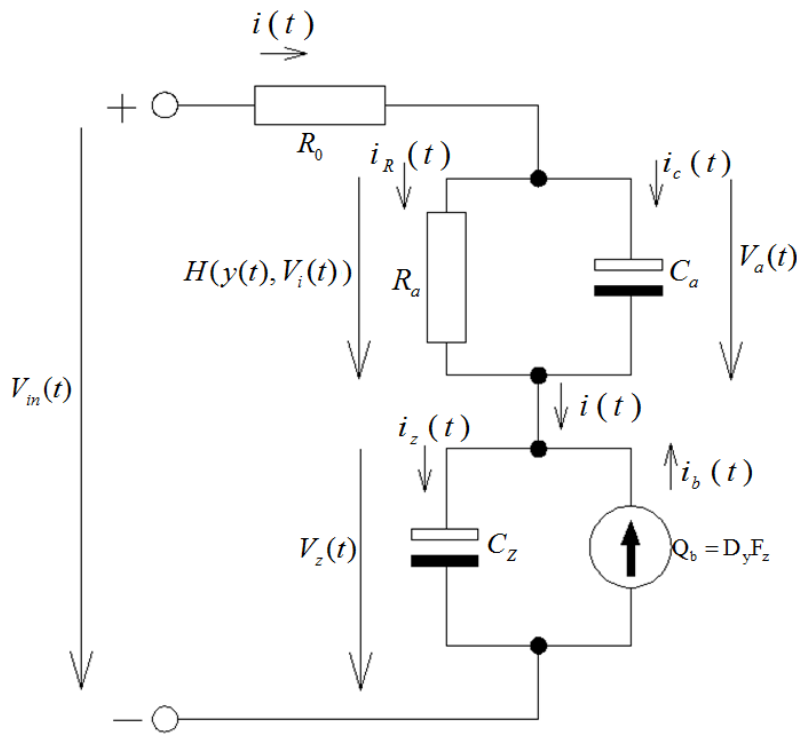

Figure 3: Scheme of the electrical part of the piezo actuator

The displacement ratio is calculated considering the surface quotient between the piezo (radius $=40 \mathrm{~mm}$ ) and the servo piston $($ radius $=4 \mathrm{~mm})$ :

$$
i_{W e g}=\frac{A_{1}}{A_{2}} .
$$

The oil compressibility is comparable with Hook's law of the material technique [13]. In [13] the concept

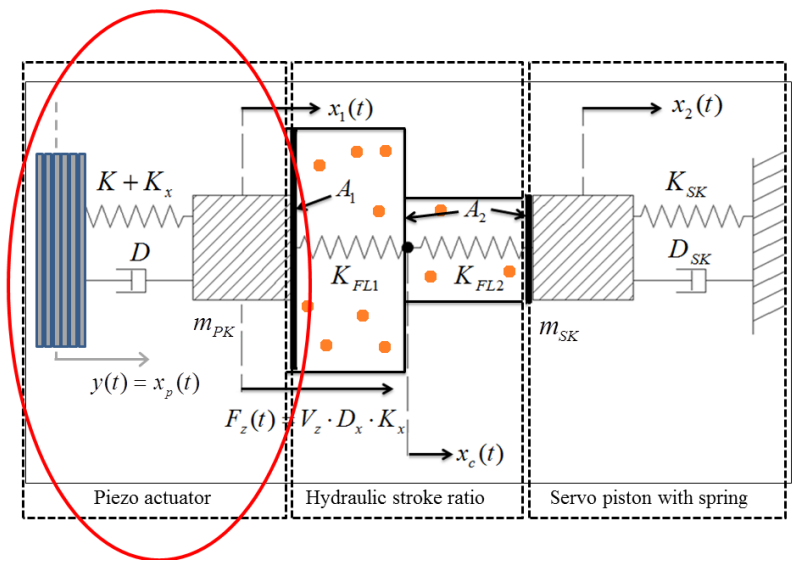

Figure 4: Mass spring model of the piezo servo piston actuator

of a liquid spring is introduced and the fluid compressibility is modelled using an elasticity factor. Considering [13], the coefficient of the liquid spring coefficient $K o_{F L}$ in a pressure form is calculated using the following expression:

$$
K o_{F L}=\frac{V_{0}}{\Delta V(t)} \Delta p(t),
$$

in which $V_{0}$ represents the total volume in the chamber. $\Delta V(t)$ is the compressed volume because of the acting force which generates a pressure difference equal to $\Delta p(t)$, see [13]. As shown in Fig. 4, two surfaces $A_{1}$ and $A_{2}$ play a role in the hydraulic ratio displacement. This ratio states the steady-state gain position factor of this part of the actuator. From Fig. 4 it is possible to observe that the model consists of two hydraulic cylinders. The forces at the connecting surfaces of both cylinders are calculated through the following product:

$F_{A 1}(t)=A_{F 1} K o_{F L 1} x_{1}(t)=\frac{A_{1}}{A_{1}+A_{2}} K o_{F L 1} x_{1}(t)$,

and

$$
F_{A 2}(t)=A_{F 2} K o_{F L 2} x_{2}(t)=\frac{A_{2}}{A_{1}+A_{2}} K o_{F L 2} x_{2}(t) .
$$

\subsection{Hydraulic Structure}

For constant pressures, the volumetric flow $Q_{t h}(t)$ of the valve drive is proportional to the length of the opening slit that equals $x_{2}(t)-\bar{x}_{2}$. Considering

$$
Q_{t h}(t)=\left(x_{2}(t)-\bar{x}_{2}(t)\right) K_{S P}
$$


with $K_{S P}$ which represents a parameter depending on the pressure and $\bar{x}_{2}(t)$ represents the initial servo piston position at which corresponds a $Q_{t h}(t)=0$. In Fig. 5 a possible model of its hydraulic part are shown as proposed in [13]. The model was presented in [13] but in a linear approximation form which is very often used in industrial applications.

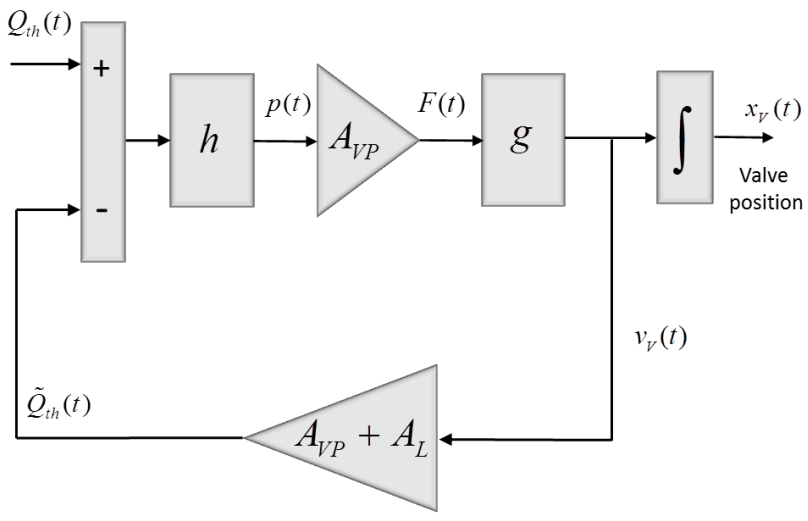

Figure 5: Block diagram structure of the hydraulic part of the actuator

$$
\dot{x}_{V}(t)=K_{H} Q_{t h}(t),
$$

where $\dot{x}_{V}(t)$ represents the velocity of the valve and $Q_{t h}(t)$ the volumetric flow. $K_{H}$ represents a physical constant which does not depend on the pressure $p(t)$ and according to [13] it is expressed as follows:

$$
K_{H}=\frac{V_{H} V_{M} A_{V P}}{1+V_{H} V_{M} A_{V P}\left(A_{V P}+A_{L}\right)},
$$

where, according to Fig. 5, $V_{H}$ is the steady-state parameter between $p(t)$ and $\tilde{Q}_{t h}(t)-Q_{t h}(t)$ and $V_{H}$ is the steady-state parameter between force and the velocity of the valve. More in depth, $K_{H}$ represents the closed loop steady-state gain of the scheme of Fig. 5, see [13]. Equation (6) states the steady state condition of the hydraulic valve system. In fact, Eq. (7) represents the steady state constant of the linear system proposed in [13] as a simplified model of the hydraulic valve system which consists of a transfer function between variables $Q_{t h}(t)$ and $x_{V}(t)$.

\section{Control Strategy}

In Fig. 6 the adopted control scheme is shown. The control scheme presents a feed-forward action to compensate the steady state error because of the absence of the integral action in the controller. Together with the feed-forward action a repetitive control algorithm is used because of the presence of a periodic signal to be tracked. It is known that the control loop can need to be stabilised and therefore a stabilising PD controller is considered in the loop.

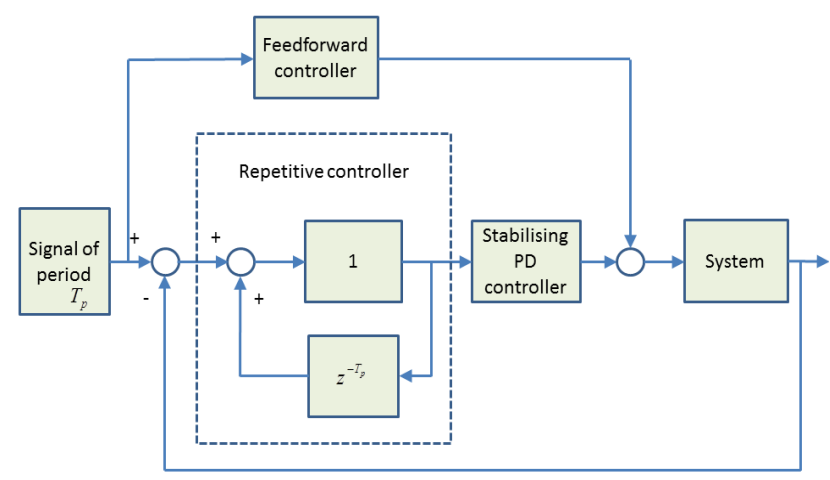

Figure 6: Block diagram of the control structure

\subsection{Feed-forward Control}

Thanks to modelling approximations described above, the inversion of the system described in Eq. (6) is as follows:

$$
Q_{t h}(t)=K_{H}^{-1} \dot{x}_{V_{d}}(t),
$$

in which $\dot{x}_{V_{d}}(t)$ represents the desired velocity of the valve. The next step is to invert Eq. (5):

$$
x_{2}(t)=K_{S P}^{-1} Q_{t h}(t)+\bar{x}_{2} .
$$

The steady-state feed-forward control can be summarized as follows: being $F_{z}(t)=F_{A 1}(t)=$ $D_{x} K_{x} V_{\text {inff }}(t)$ and $p(t)=\frac{F_{A 2}(t)}{A 2}=\frac{F_{A 1}(t)}{A 1}$, then $F_{A 2}(t)=\frac{A_{2}}{A_{1}} F_{z}(t)$ and considering Eq. (4), then $A_{F 2} K_{F L 2} x_{2}(t)=\frac{A_{2}}{A_{1}} D_{x} K_{x} V_{i n f f}(t)$. It is straightforward to obtain the following relation:

$$
V_{\text {inff }}(t)=\frac{A_{F 2} K_{F L 2}\left(K_{S P}^{-1} K_{H}^{-1} \dot{x}_{V d}(t)+\bar{x}_{2}\right)}{\frac{A_{2}}{A_{1}} D_{x} K_{x}} .
$$

\section{Experimental Results Using Repet- itive Control}

According to the standard control scheme represented in Fig. 6 some measurements were done. It is known 
that a repetitive controller is characterised by the following transfer function:

$$
G_{r}(s)=\frac{1}{1-e^{-T_{p} s}} .
$$

Parameter $T_{p}$ represents the period of the periodical signal to be tracked. Normally, the repetitive controller is realised using a discrete technique and Eq. (11) becomes as follows:

$$
G_{r}(z)=\frac{1}{1-z^{-T_{p}}} .
$$

Figure 7 shows the measured results of pressure inside the stroke ratio, Fig. 8 shows the position measurements, Fig. 9 shows the position of the servo structure and finally Fig. 10 shows the trajectory position of the intake vale for $3000 \mathrm{rpm}$. A detailed scheme of the repetitive control idea is shown in Fig. 6. Measured results, in an experimental setup using a dSPACE system to implement the control and the fault detection structure, confirm that the inversions described in 3.1 can be used as an effective feed-forward control for the presented hybrid actuator.

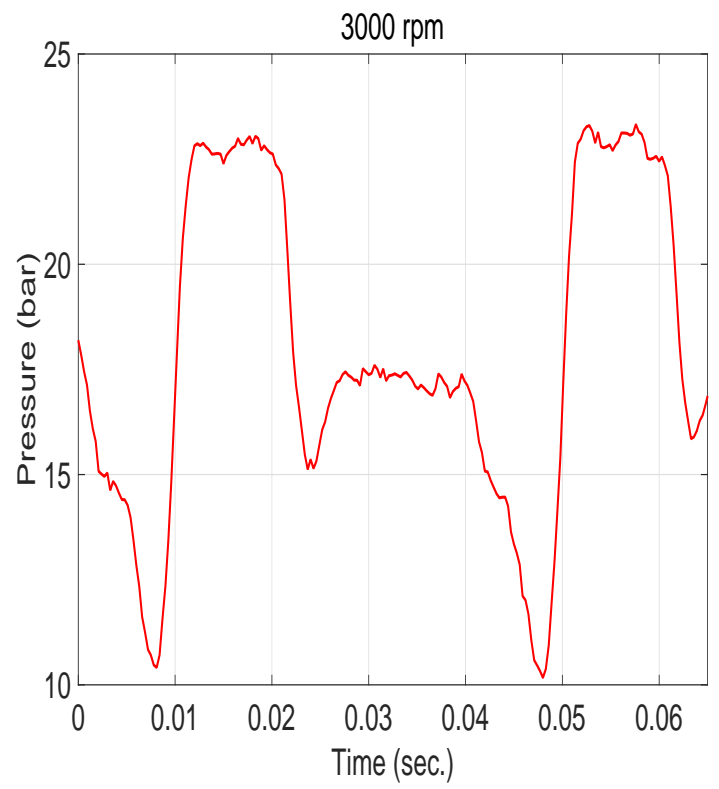

Figure 7: Pressure inside the stroke ratio

\section{Conclusion and Outlook}

The paper deals with modelling and control of a hybrid actuator. Particular attention is paid to a hydraulic spring model of the ratio displacement which represents the hydraulic part of the mechanism. A repetitive controller is applied to track a periodical signal

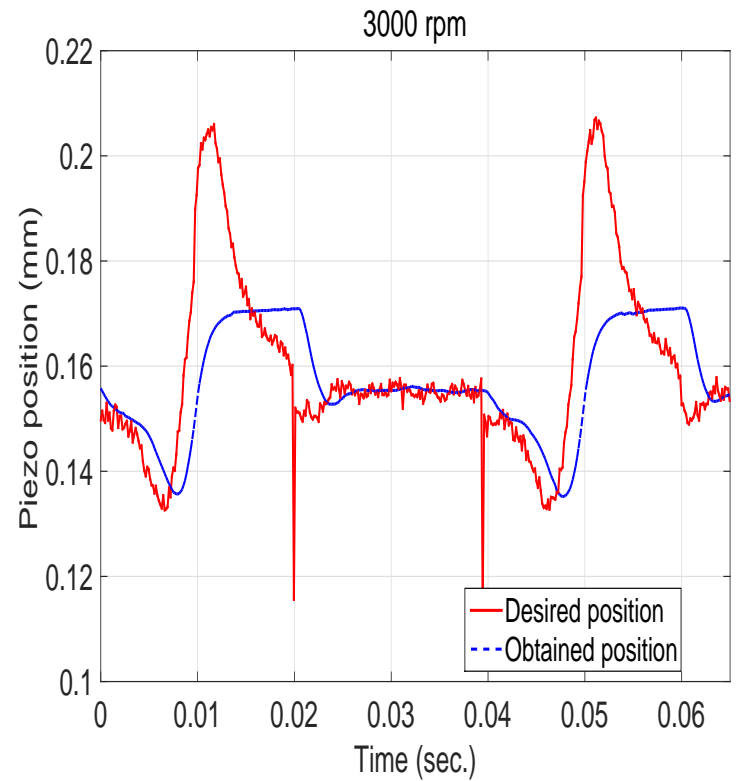

Figure 8: Position of the piezo

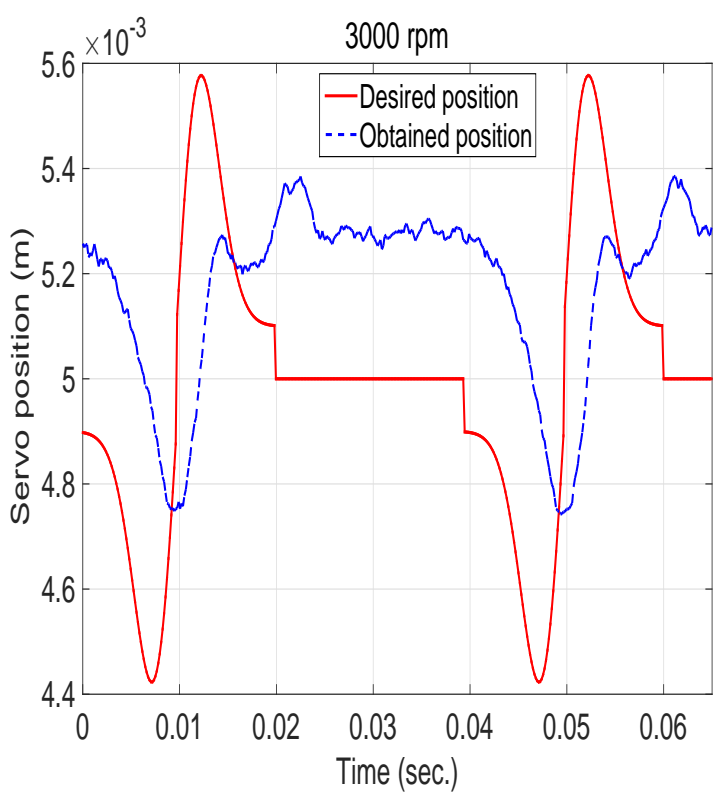

Figure 9: Position of the servo piston

together with a PD-controller which is devoted to stabilise the feedback control loop. Measured results are presented to demonstrate the effectiveness of the proposed method. Future advancements of this work can include a detailed friction model of the mechanical system and its control using Sliding Mode Control as proposed in [14] and in [15]. Moreover, in order to reduce the number of sensors an implementation of a Kalman Filter can be considered as proposed in [16].

Acknowledgements: The author wants to acknowledge Lucas Köhler, Guido Bergholz and Robert Zylla 


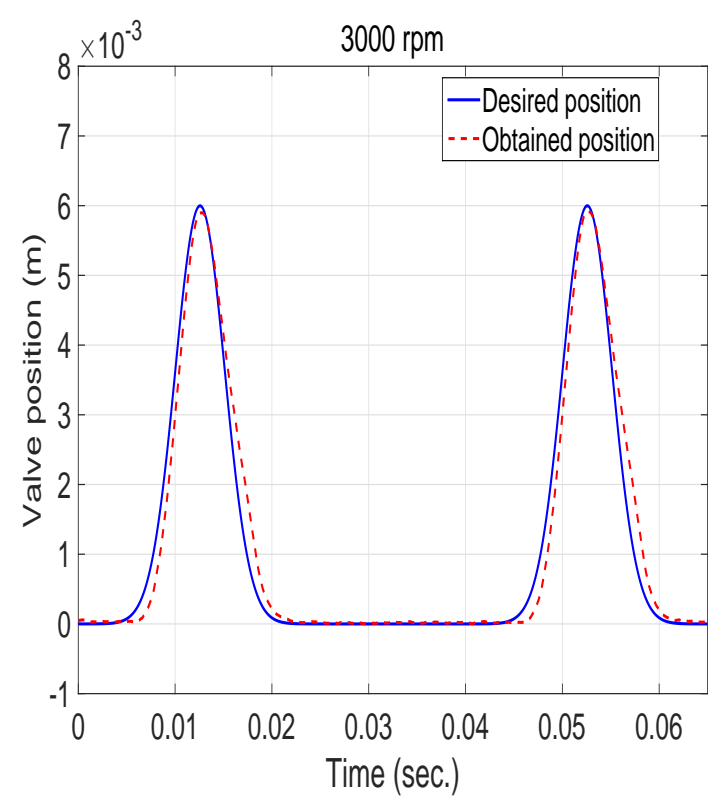

Figure 10: Position of the valve

of Ostfalia University of Applied Sciences for the collaboration in doing the measurements. The paper could not be accomplished without them.

\section{References:}

[1] D. Croft, G. Shed, and S. Devasia. Creep, hysteresis, and vibration compensation for piezoactuators: Atomic force microscopy application. Transactions of the ASME Journal of Dynamic Systems, Measurement, and Control, 123(1):3543, 2001.

[2] M.S. Rana, H.R. Pota, and I.R. Petersen. Nonlinearity effects reduction of an afm piezoelectric tube scanner using mimo mpc. Transactions on Mechatronics, 20:1458-1469, October 2014.

[3] F. Todeschini, M. Corno, G. Panzani, S. Fiorenti, and S.M. Savaresi. Adaptive cascade control of a brake-by-wire actuator for sport motorcycles. Transactions on Mechatronics, 20:1310-1319, August 2014.

[4] J.-M. Rodriguez-Fortun, J. Orus, J. Alfonso, F.B. Gimeno, and J.A. Castellanos. Flatness-based active vibration control for piezoelectric actuators. Transactions on Mechatronics, 18:221229, September 2012.

[5] S. Wilcox and S. Devasia. Stability of velocity control for a piezoelectric stepper. Transactions on Mechatronics, 20:910-923, June 2014.
[6] M. Rakotondrabe, I.A. Ivan, S. Khadraoui, P. Lutz, and N. Chaillet. Simultaneous displacement/force self-sensing in piezoelectric actuators and applications to robust control. Transactions on Mechatronics, 20:519-531, January 2014.

[7] B.A. Francis and W.M. Wonham. The internal model principle for linear multivariable regulators. Journal of Applied Mathematics and Optimization, 2(4):170-194, 1975.

[8] H.L. Trentelman and J.C. Willems. Essays on Control: Perspectives in the Theory and its Applications. Birkhäuser, Boston, Basel, Berlin, 1993.

[9] P. Mercorelli and N. Werner. A model of a servo piezo mechanical hydraulic actuator and its regulation using repetitive control. In Proc. of the International Conference on Advanced Intelligent Mechatronics (AIM), 2014 IEEE/ASME, pages 186-191, Besancon, 2014.

[10] P. Mercorelli and N. Werner. An adaptive resonance regulator design for motion control of intake valves in camless engine systems. IEEE Transactions on Industrial Electronics, 64(4):3413-3422, 2017.

[11] P. Mercorelli and N. Werner. A hybrid actuator modelling and hysteresis effect identification in camless internal combustion engines control. International Journal of Modelling, Identification and Control, 21(3):253-263, 2014.

[12] Y.-C. Yu and M.-K. Lee. A dynamic nonlinearity model for a piezo-actuated positioning system. In Proceedings of the 2005 IEEE International Conference on Mechatronics, ICM $10^{\text {th }}$ $12^{\text {th }}$ July, Taipei, 2005.

[13] H. Murrenhoff. Servohydraulik. Shaker Verlag, Aachen, 2002.

[14] T. Ferch and P. Mercorelli. Vertical dynamics description and its control in the presence of nonlinear friction. WSEAS Transactions on Systems, 18:198-212, 2019.

[15] K.-C. Schwab, L. Schräder, P. Mercorelli, and J.T. Lassen. Sliding mode and model predictive control for inverse pendulum. WSEAS Transactions on Systems and Control, 14:190-195, 2019.

[16] J.T. Lassen and P. Mercorelli. Tuning kalman filter in linear systems. WSEAS Transactions on Systems and Control, 14:209-212, 2019. 